\title{
Fabrication of micro rods by twin-mirroring- wire tangential feed micro electrical discharge grinding
}

\author{
Yanqing Wang, Mattia Bellotti, Jianyu Jia, Zan Li, Shengqiang Yang, Jun Qian, \\ Dominiek Reynaerts \\ ${ }^{a}$ Key Laboratory of Precision Machining of Shanxi Province, College of Mechanical and Vehicle Engineering, Taiyuan University of Technology, Wanbailin District, \\ Taiyuan, Shanxi, China \\ ${ }^{\mathrm{b}}$ Department of Mechanical Engineering, KU Leuven and Member Flanders Make, Leuven, Belgium
}

\section{ABSTRACT}

In micro electrical discharge machining (micro-EDM), the precision fabrication of cylindrical micro rods is difficult to achieve with a high processing efficiency. In order to overcome this challenge, this paper proposes a new processing method, which is denoted as twin-mirroring-wire tangential feed micro electrical discharge grinding (TMTF-WEDG). The machining principle, characteristics, and realization of the new method are firstly introduced. Then, the advantages of TMTF-WEDG in terms of machining efficiency and accuracy are demon-strated. The experimental results have shown that the machining efficiency can be increased to more than $70 \%$ in comparison with conventional tangential-feed wire electrical discharge grinding. It has also been proved that a minimum removal of material corresponding to a reduction of less than $1 \mu \mathrm{m}$ in the diameter of a micro rod can be obtained by TMTF-WEDG. This considerably helps in improving the accuracy and repeatability of the machining process. A deviation of less than $1 \mu \mathrm{m}$ on the diameter of a micro rod has been obtained in a length range of $800 \mu \mathrm{m}$. The process repeatability in machining five micro rods has been established to be below $2 \mu \mathrm{m}$. The proposed method is therefore of great significance for improving the machining efficiency and ensuring a high precision in the shaping process of cylindrical micro rods.

\section{Introduction}

In recent years, the demand for micro-scale components and products has increased rapidly, particularly in the fields of electronics, communications, optics, avionics, medicine, and automobiles [1]. This has resulted in considerable research and technological advancements in the field of micro machining processes. In this context, micro electrical discharge machining (micro EDM) has been established as a reliable option for fabricating micro structures of high aspect ratio, thanks to the non-contact nature of its material removal process [2]. However, unlike conventional macro-scale EDM, the application of micro EDM is hindered by some limitations in handling and preparation of the tool electrodes, as well as in planning of the machining operations [3].

In micro-EDM, cylindrical micro rods are typically used as tool electrodes for drilling micro holes or milling micro structures. These micro rods are usually adjusted on-the-machine to the desired diameter and length, starting from commercially available rods of standard size. This means that the efficiency and accuracy in fabricating the micro rods directly affect the efficiency and accuracy of the whole process for machining the micro holes and structures. Therefore, the realization of highly efficient and precise methods for fabricating micro rods is of great significance for expanding the field of application of the micro EDM technology.

Nowadays, block electrode discharge grinding (BEDG) and wire electrode discharge grinding (WEDG) are the typical technologies for fabricating micro rods in industrial applications. In BEDG, due to the wear of the block electrode, it is rather difficult to control the dimensional accuracy of the micro rods. Block electrodes with high electrical resistivity have been used to reduce the block wear [4]. A horizontal moving BEDG method was proposed by Hourmand et al. [5] in order to increase the area on the block, which is used for the discharging process, and therefore reduce the effects of the block wear on the machining accuracy. However, this strategy asks for re-machining of the surface of the block electrode before BEDG. This is costly and time-consuming. WEDG, which was proposed by Masuzawa in 1985 [6], has been one of the most significant developments in micro EDM. In this process, a 
moving wire is used to avoid the problem of electrode wear. Therefore, accurate machining of micro rods can be achieved. A tangential feed method (TF-WEDG) was developed by Zhang et al. [7] in order to reduce the effects of the positioning errors during the fabrication process, and therefore improve the diameter accuracy. The experimental results have shown that a diameter uniformity of less than $1 \mu \mathrm{m}$ can be achieved by TF-WEDG, while the machining accuracy in fabricating several micro rods is less than $2 \mu \mathrm{m}$. Li et al. developed a novel active supplying wire-electro discharge grinding (AS-WEDG) device to machine micro rod with high accuracy as $1.5 \mu \mathrm{m}(\varnothing 42.6 \mu \mathrm{m}, 900 \mu \mathrm{m}$ length) and $2.5 \mu \mathrm{m}$ (Ø $40.3 \mu \mathrm{m}, 2000 \mu \mathrm{m}$ length) [8]. But the moving wire electrode can cause fluctuations in the front edge of the machining area, which may be the cause of the diameter deviation. Zero infeed and finishing using a stationary wire electrode have been also proposed as solutions to eliminate some of the inherent errors of WEDG [9].

The accuracy and efficiency of electrical discharge grinding processes can also be increased by using a second sacrificial electrode. Qingfeng et al. [10] proposed electrical discharge grinding using two block electrodes (EDG-TBE). In this method, the narrow slit between two block electrodes is used to control the micro rod diameter. EDG-TBE was proved to be a more efficient machining method than BEDG, allowing for an average error below $2 \mu \mathrm{m}$ between the target and machined diameter of the micro rods. Naotake et al. [11] developed a novel processing method by EDM, in which a micro rod is driven along the midline of the slit between two electrically isolated metal plates. The experimental results have shown that micro rods of $20 \mu \mathrm{m}$ diameter can be machined by this method. To improve the processing efficiency of WEDG, Sheu [12,13] proposed a twin-wire WEDG system, which combines a transistor and a resistor-capacitor (RC) power supplies for fabricating micro rods by simultaneously carrying out rough and finish machining. The two wire electrodes are arranged on two different levels in this system. Compared to conventional WEDG using a single wire electrode, the twin-wire WEDG system can reduce the machining time by about $60 \%$.

Based on the abovementioned studies, two main conclusions can be drawn. It can be concluded that the use of a tangential feed strategy is beneficial either for compensating the electrode wear or reducing the effects of the positioning errors of the machine tool. In WEDG, a tangential strategy can also be used to achieve a minimum material removal of less than $1 \mu \mathrm{m}$ in the diameter of a micro rod [8]. This is advantageous for precise control of the micro rod diameter and thus improve the repeat processing accuracy of several micro rods. Second, it can be concluded that the machining efficiency can be increased by using two sacrificial electrodes. The use of two electrodes also allows for the application of two power supplies, which is a way to even further improve the processing efficiency. Furthermore, the narrow slit between the two electrodes facilitates the precise control of the diameter of the processed micro rods.

In order to fabricate micro rods having a high diameter uniformity and a high repeat processing accuracy with a high processing efficiency, this paper proposes a new method, which combines the use of two wire electrodes and a tangential feed strategy. This method is denoted as twin tangential feed wire electrical discharge grinding (TMTF-WEDG). In TMTF-WEDG, the narrow slit between two wire electrodes is used to control the diameter of the processed micro rods. Two power supplies are used to maximise the machining efficiency. Section 2 introduces the principle of the new processing method and analyses characteristics of TMTF-WEDG and the machining efficiency. In Section 3, the micro EDM system used for the experimental activities is presented. Section 4 discusses the experimental results, demonstrating the feasibility and advantages of the proposed processing method.

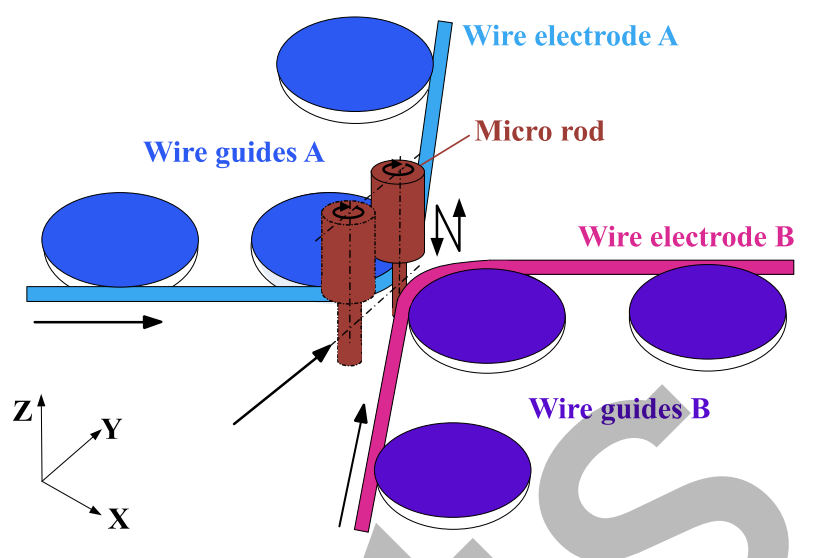

Fig. 1. Schematic diagram of the TMTF-WEDG process. The black arrows denote the feed of the micro rod and wire electrodes.

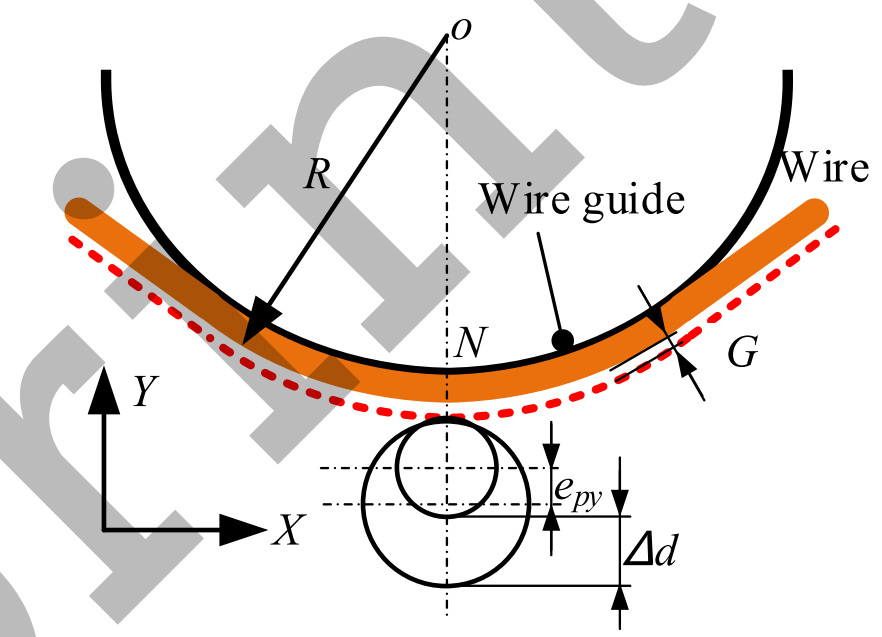

Fig. 2. The effect of Y-axis positioning error on the micro rod diameter.

\section{Twin -mirroring-wire tangential feed electrical discharge grinding}

\subsection{Principle of TMTF-WEDG}

The principle of the proposed TMTF-WEDG method is shown in Fig. 1. Two electrically insulated wire electrodes are used. The two wires are located in the same X-Y plane. This is different from the WEDG method described in Ref. [12,13], in which one of the two wires lies on a higher horizontal plane with respect to the other wire.

The movements of the two wire electrodes and micro rod during TMTF-WEDG processing are illustrated in Fig. 1. The movement of the micro rod is the combination of three motions: (i) the motion along the midline of the two wire electrodes in tangential direction with respect to the wire guides, which determines the diameter of the micro rod, (ii) the reciprocating motion along the Z-axis of the machine tool, which determines the length of the micro rod, and (iii) the rotation around the axis of the micro rod. The wire electrodes are meant to move at a constant speed during rough and semi-finishing machining. This allows for compensation of the wear of the wire electrodes. In finishing machining, in order to reduce or eliminate the influence of the vibrations of the wire electrodes on the diameter uniformity of the micro rod, the wire electrodes can move at a lower speed or even be stationary.

To improve the repeat processing accuracy of several micro rods fabricated by radial-feed WEDG shown in Fig. 2, the most important is to ensure the repeated positioning accuracy of the micro rod in $\mathrm{Y}$ direction, this is because the diameter error of machined micro rod is twice of the 
a)

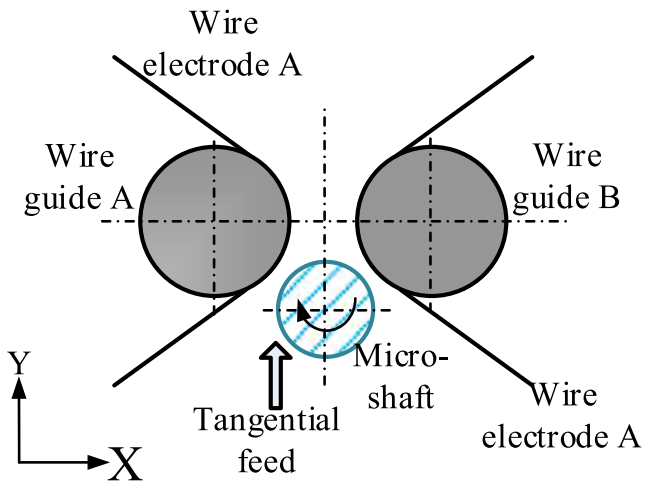

b)

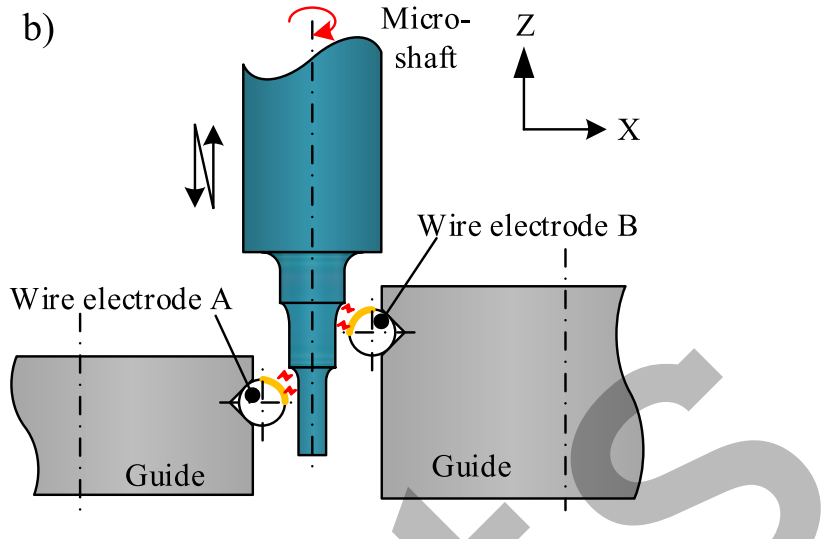

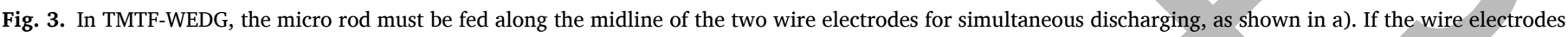
are not coplanar as in b), the machining efficiency is not maximised.

positioning error of Y-axis [7]. But, for the TMTF-WEDG, it just needs to ensure that the micro rod is located on the midline of the twin-wire electrodes during the processing, and the formed narrow slit by twin-wire electrodes can be used to control the micro rod diameter. Keeping the micro rod on the midline of the twin-wire electrodes has been well achieved, which is introduced in section 4.1. Therefore, the new machining method reduces the requirement for repeated positioning accuracy of the machine tool in the $\mathrm{Y}$ direction.

The new method takes advantages of the high removal resolution (the minimum thickness of micro rod caused by unit feed distance in tangential direction) due to the use of the tangential feed strategy, the precise diameter of micro rod can be achieved by the tangential feed of the micro rod to a certain position. During the TFTM-WEDG, as long as the micro rod is located on the midline of the twin-wire electrodes, then the micro rod diameter can be controlled by the controlling of the tangential feed distance. And the closer to the minimum slit width, the smaller the removal layer thickness of the micro rod. Where the slit width is the smallest, the diameter of the processed micro rod and the removed layer thickness caused by the positioning error of the micro rod are both the smallest (Discussed in section 2.2.2). Therefore, this is advantageous for improving the repeat processing accuracy of the small diameter micro rods. In other words, even if there is a large positioning error of machine tool in tangential feed direction, the removal layer thickness of the micro rod due to the positioning error is also very small. This helps to improve the repeat processing accuracy of the micro rods and reduces the requirements for the positioning accuracy in the tangential direction of the machine tool.

In addition, thanks to the use of two electrically insulated wires, the proposed method allows for the use of a dual power supply. This offers

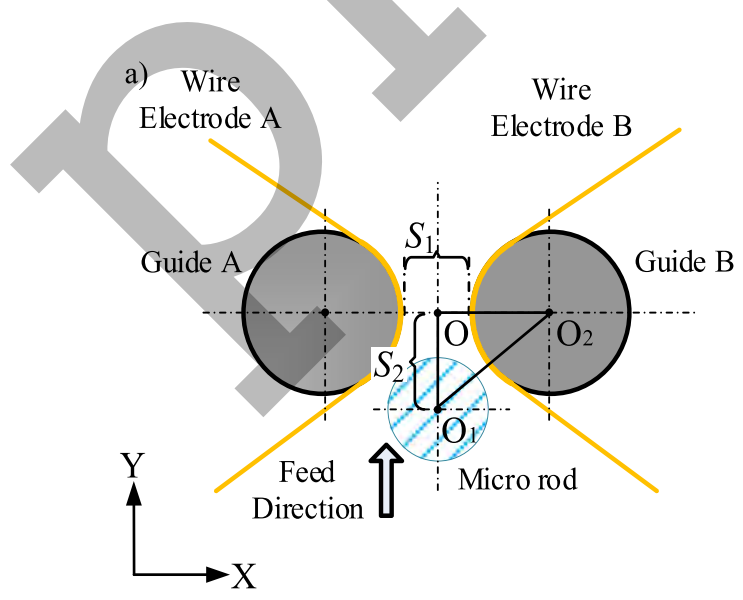

the possibility to potentially double the processing efficiency.

\subsection{Theoretical analysis of TMTF-WEDG}

\subsubsection{Machining efficiency}

In order to widen the application field of WEDG in modern fabrication industries, it is important to improve its machining efficiency. The TMTF-WEDG method proposed in this paper uses two electricallyinsulted wire electrodes, which allow for using two power supply systems.

If the electric discharges simultaneously occur between the two wire electrodes and the micro rod, the processing efficiency can be improved in comparison to the traditional WEDG process using a single wire electrode. Two conditions need to be met in order to ensure the simultaneity of the electric discharges. First, the micro rod needs to be fed along the symmetrical midline of the two wire electrodes (Fig. 3a). Second, the two wire electrodes should be in the same $\mathrm{X}-\mathrm{Y}$ plane during the reciprocating motion of the micro rod. The reason behind the need of this latter condition can be explained using the schematic illustration in Fig. 3b. If the two wires electrodes were not coplanar, there would be a time period during the downward motion of the micro rod in which electrical discharges would only occur between the upper wire and the micro rod. And the duration of one-sided discharge is the height difference between the two-wire electrodes divided by the Z-axis feed speed. Therefore, the machining efficiency would not be maximised.

\subsubsection{Control of the micro rod diameter}

The diameter of a micro rod after TMTF-WEDG processing can be calculated with the support of the schematic illustrations in Fig. 4a-b.

Fig. 4. Schematic diagram for calculating the diameter of a fabricated micro rod. a) Top view; b) Main view.

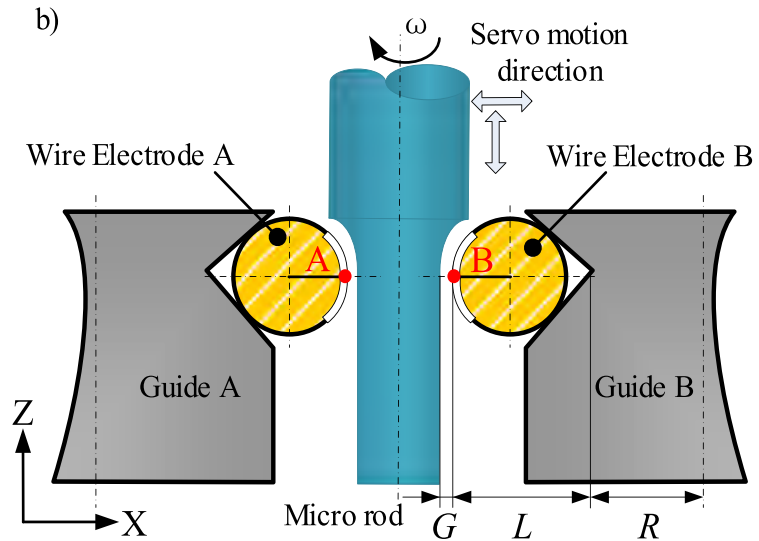




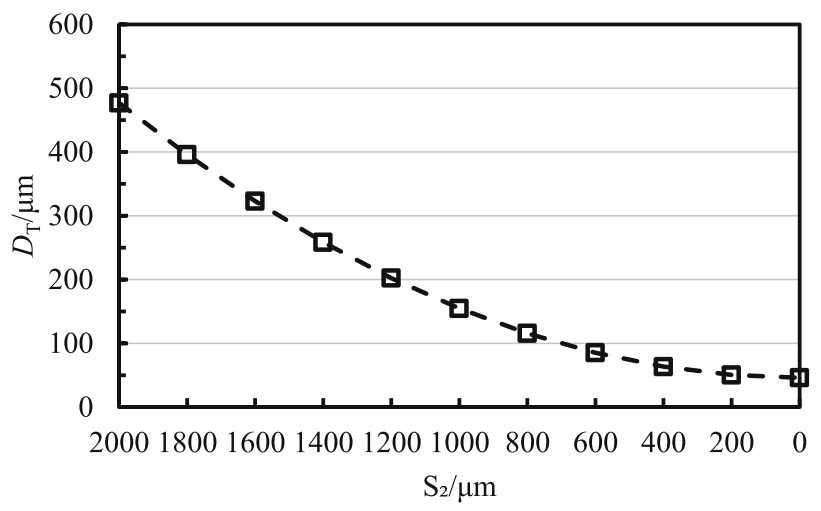

(a) $0<S_{2} \leq 2000 \mu \mathrm{m}$

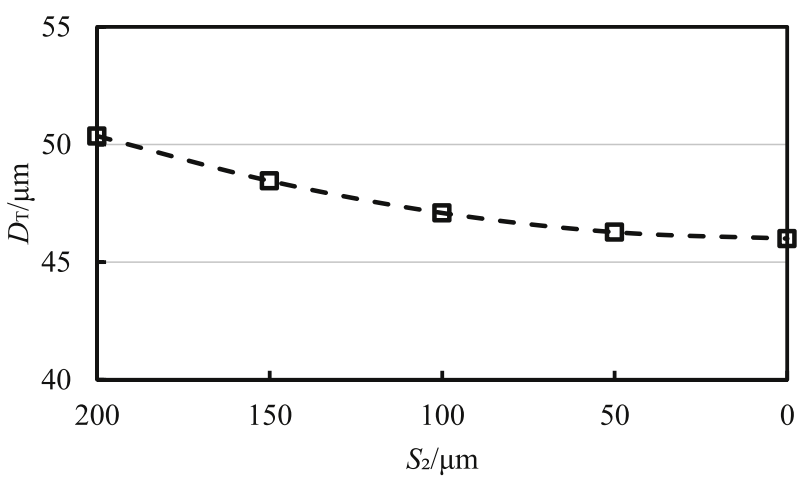

(b) $0<S_{2} \leq 200 \mu \mathrm{m}$

Fig. 5. Variations to the diameter $D_{T}$ of a micro rod when varying the control variable $S_{2}$. The graph is obtained by calculation according to Eq. (4) with considering the following conditions: $S_{1}=50 \mu \mathrm{m}, G=2 \mu \mathrm{m}$, and $R_{A}=9000 \mu \mathrm{m}$.

Based on these illustrations, the following equations can be written:

$O O_{1}^{2}+O O_{2}^{2}=O_{1} O_{2}^{2}$

$O O_{1}=S_{1} / 2+G+R_{A}$

$O_{1} O_{2}=D_{T} / 2+G+R_{A}$

where:

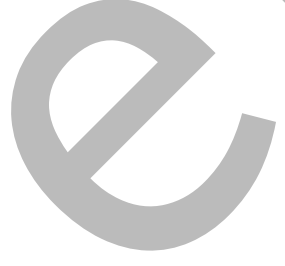

(2)

$D_{T}$-diameter of the micro rod;

$S_{1}$-distance between the two apexes at the wire electrodes;

$S_{2}$-distance between the final position of the micro rod and the point A;

$G$ - discharge gap;

$R_{A}$-distance between the apex of the wire electrode and the centre of the wire guide. This distance depends on the sizes of the wire guides and wire electrodes.

Substituting Eq. (2) and Eq. (3) into Eq. (1), the diameter of a micro $\operatorname{rod}\left(D_{T}\right)$ can be expressed as:

$D_{T}=2\left(\sqrt{\left(S_{1} / 2+R_{A}\right)^{2}+S_{2}^{2}}-G-R_{A}\right)$

$\frac{\mathrm{d} D_{T}}{\mathrm{~d} S_{2}}=\frac{2 S_{2}}{\sqrt{\left(S_{1}+R A\right)^{2}+S_{2}^{2}}}$

It can be seen from Eq. (4) that the diameter of a micro rod depends

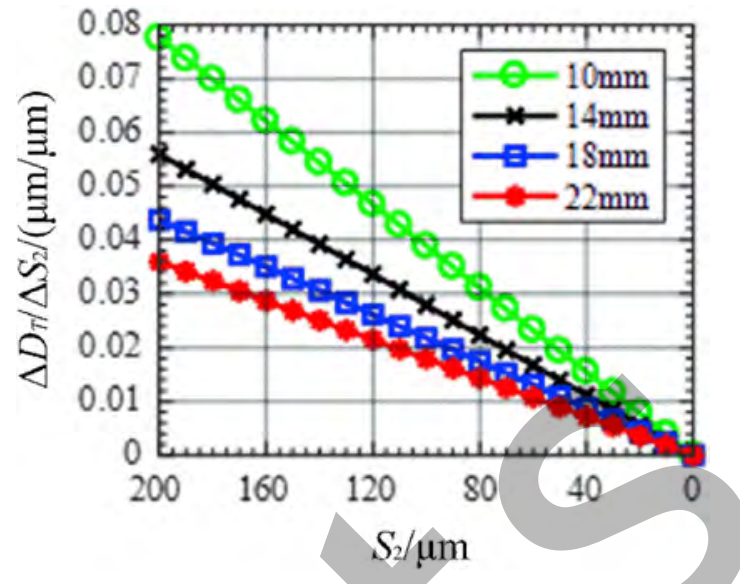

Fig. 6. Reduction rate in the diameter of a micro $\operatorname{rod}\left(\Delta D_{T} / \Delta S_{2}\right)$ with respect to the feed distance $\left(S_{2}\right)$ at different values of the diameter of the wire guides calculating according to Eq. (5).

on four variables: $S_{1}, R_{A}, S_{2}, G$. As shown in Fig. 4, the diameter deviation and vibration of the wire electrode lead to variations of $R_{A}$, and thus also directly affect the diameter of the micro rod. The discharge gap $G$ is mainly determined by the discharge energy and the debris concentration in the discharge area. Considering that a low open voltage, small discharge energy, and good flushing conditions are relatively easy to achieve, the discharge gap is relatively small and its fluctuation can be limited. This helps in improving the uniformity and repeatability of the diameter of the machined micro rods. The other two variables determining the diameter of a micro rod are $S_{1}$ and $S_{2}$. These two variables are selected as the input variables for controlling the diameter of a micro rod.

Fig. 5 shows how the diameter of a micro rod varies with $S_{2}$ in the range between 0 and $2000 \mu \mathrm{m}$, under the following conditions: $S_{1}=50$ $\mu \mathrm{m}, G=2 \mu \mathrm{m}$, and $R_{\mathrm{A}}=9000 \mu \mathrm{m}$. The rules are obtained by calculation according to Eq. (4). When the value of $S_{2}$ is relatively high, it can be noticed that the diameter $D_{\mathrm{T}}$ decreases sharply. With the decreasing of $S_{2}$, the reduction in diameter is more gradual. The variation of $D_{\mathrm{T}}$ when $S_{2}$ is in the range $0-200 \mu \mathrm{m}$ is shown in the zoom-in of Fig. 5 . It can be seen that when $S_{2}$ is reduced from $200 \mu \mathrm{m}$ to $0 \mu \mathrm{m}$, the reduction in diameter of a micro rod is only $4 \mu \mathrm{m}$. Therefore, a fine control of the thickness of material removed from a micro rod can be achieved by controlling $S_{2}$.

Fig. 6 shows the rate of the diameter change of the micro rod corresponding to per unit tangential feed distance with respect to $S_{2}$, when four different levels of the diameter of the wire guides are considered: $10 \mathrm{~mm}, 14 \mathrm{~mm}, 18 \mathrm{~mm}$, and $22 \mathrm{~mm}$. It can be noticed that the reduction in diameter of a micro rod linearly decreases with $S_{2}$, and it approaches 0 when the position the micro reaches the point $O$. Therefore, in applications requiring a tight tolerance on the micro rod diameter, the ratio $\Delta D_{T} / \Delta S_{2}$ should be minimised so as that the final position of the micro rod should be as close as possible to the point $\mathrm{O}$.

\section{Experimental setup}

\subsection{Micro EDM system}

Fig. 7 shows the developed micro EDM system for TMTF-WEDG processing. The system consists of a micro EDM machine, a TMTFWEDG unit, a 3-axis control system, a dual RC power supply unit, a dielectric unit, and a CCD on-machine vision system. The X, Y, Z axes of the micro EDM machine are driven by AC servo motors and leadscrews, with a step feed of $0.1 \mu \mathrm{m}$. The TMTF-WEDG unit is integrated into the micro EDM machine. This allows for on-machine fabrication of micro rods, which can be used as tool electrodes for micro EDM milling or 


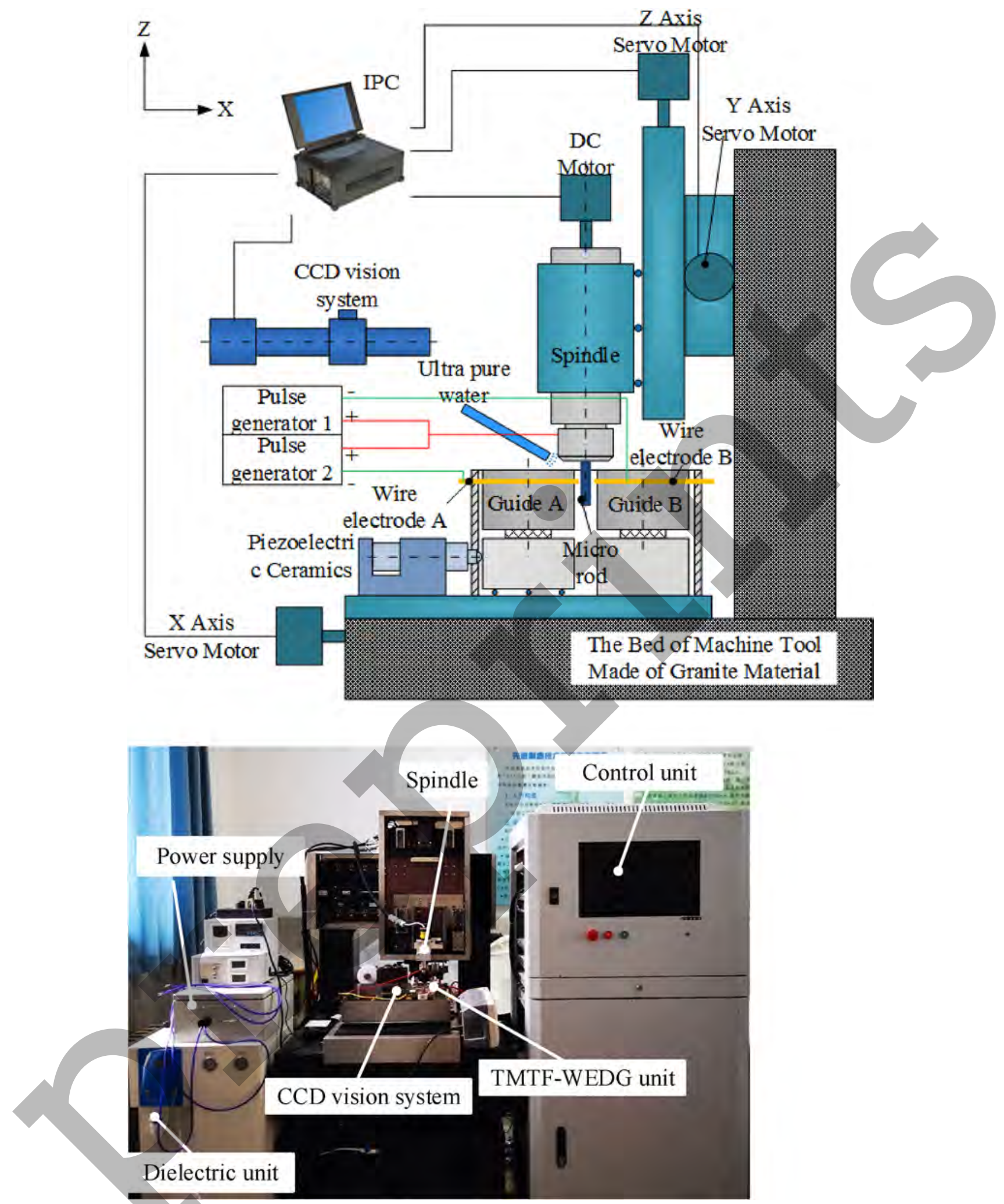

Fig. 7. Schematic diagram (top) and picture (bottom) of the developed micro EDM system.

drilling.

The dielectric unit includes a water reservoir, a filtration system, and a deionization system, which is used to ensure the water conductivity level. The CCD on-machine vision system is used to measure the diameter of the micro rods after TMTF-WEDG processing. The resolution of the CCD camera is $2048 \times 2448$ pixels, and a 10x lense was used. This provided a resolution of $0.345 \mu \mathrm{m}$ in lateral and longitudinal direction.

\subsection{Power supply system}

A schematic of the two independent resistor capacitor (RC) power supplies used for TMTF-WEDG is shown in Fig. 8. The resistivity of the 


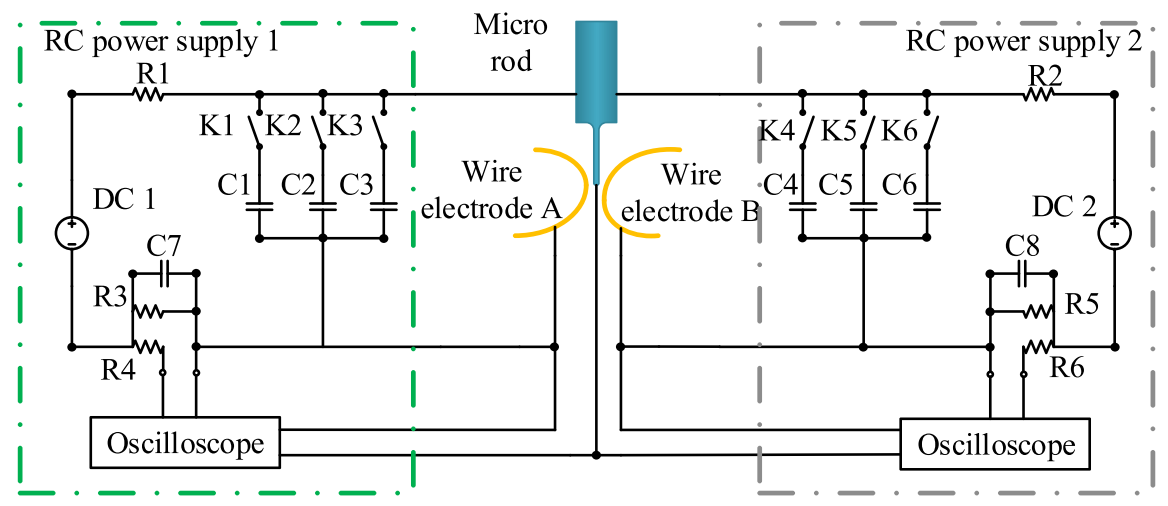

Fig. 8. RC power supplies used in the TMTF-WEDG system.

Table 1

Applied parameters and conditions for TMTF-WEDG processing.

\begin{tabular}{llll}
\hline & Roughing & Semi-finishing & Finishing \\
\hline Open voltage & $120 \mathrm{~V}$ & $100 \mathrm{~V}$ & $40 \mathrm{~V}$ \\
Capacitance & $68 \mathrm{nF}$ & $470 \mathrm{pF}$ & $47 \mathrm{pF}$ \\
Wire speed & $0.11 \mathrm{~mm} / \mathrm{s}$ & $0.11 \mathrm{~mm} / \mathrm{s}$ & $0 \mathrm{~mm} / \mathrm{s}$ \\
Spindle speed & $1200 \mathrm{rpm}$ & \\
R1/R2 & $1000 \Omega$ & \\
Dielectric fluid & Deionized water $(0.0556-0.0588 \mu \mathrm{S} / \mathrm{cm})$ & \\
Flushing pressure & $\leq 0.1 \mathrm{MPa}$ & \\
Micro rods & Brass $(\varnothing 0.5 \mathrm{~mm})$ & \\
Wire electrodes & Brass $\left(\varnothing 0.25^{\circ}{ }_{-0.002} \mathrm{~mm}\right)$ & \\
\hline
\end{tabular}

finishing, and finishing. The processing parameters applied during each stage of the machining process are shown in Table 1 . A high material removal rate is achieved during roughing by increasing the energy of the discharge pulses. Due to the relatively high discharge energy, it was found that the machined micro rods are typically characterised by a large variation in diameter along the axial direction using a single-pass machining strategy. The semi-finishing stage is therefore meant to prepare micro rods having a higher diameter uniformity in the axial direction. After semi-finishing, the diameter of the micro rods should be close to the target diameter. The purpose of finishing is mainly to decrease the diameter of a micro rod at small material removal rate in order to precisely control the diameter and gradually reach the target diameter.

The applied feeding strategy is shown in Fig. 9. The feeding in $y+$ direction is used to adjust the radial cutting depth. The feeding in $\mathrm{z}+/ \mathrm{z}$ directions is used to remove the material in the axial direction of the micro rod. It is similar to the feeding applied in a lathe machine tool.

During roughing and semi-finishing, the two wire electrodes move at a constant speed of $0.11 \mathrm{~mm} / \mathrm{s}$. This allows for compensation of the wire wear. A processing strategy involving stationary wire electrode running is adopted in finishing. Thus, the influence of the vibrations of the wire electrodes on the diameter uniformity of the micro rods is reduced or even eliminated. The tangential feeding of the micro rod as in Fig. 9 advances the discharging point to a fresh portion of the surface on the wire electrodes. The effectiveness of using this strategy can be explained as follows. As it can be seen from Fig. 6, when the micro rod is close to the narrowest point of the slit between the two wire electrodes, the diameter reduction of the micro rod in the unit feed distance of $S_{2}$ is only $0.01 \mu \mathrm{m}$. Thus, the material removal and wire wear are also very small. An image of the worn wire electrodes after TMTF-WEDG processing is shown in Fig. 10. The image was taken by means of the CCD on-machine vision system. It can be seen that the surface of the worn wire electrodes has a wavy structure, which is extremely shallow. The wavy structure is the result of the positions at which discharges between the micro rod and the two wire electrodes occur after each tangential feeding step.

\subsection{Tool setting}

The sensing contact method is used to setting the position of the micro rod. The voltage between the micro rod and the twin wire electrodes are monitored during the tool-workpiece setting up. When the micro rod contacts the wire electrodes, the gap voltage switches between high-level voltage and low-level voltage, and thus the duty cycle of the voltage waveform can be used to evaluate the contact state between the micro rod and the wire electrode. If the rotation center of the micro-rod lies on the midline of the slit, the duty cycle difference of the two tool setting waveforms should be less than a certain threshold. During the tool setting, record and count the duty cycle differences of the two voltage waveforms over a certain period. If the average value of

The machining process is divided into three stages: roughing, semi- 


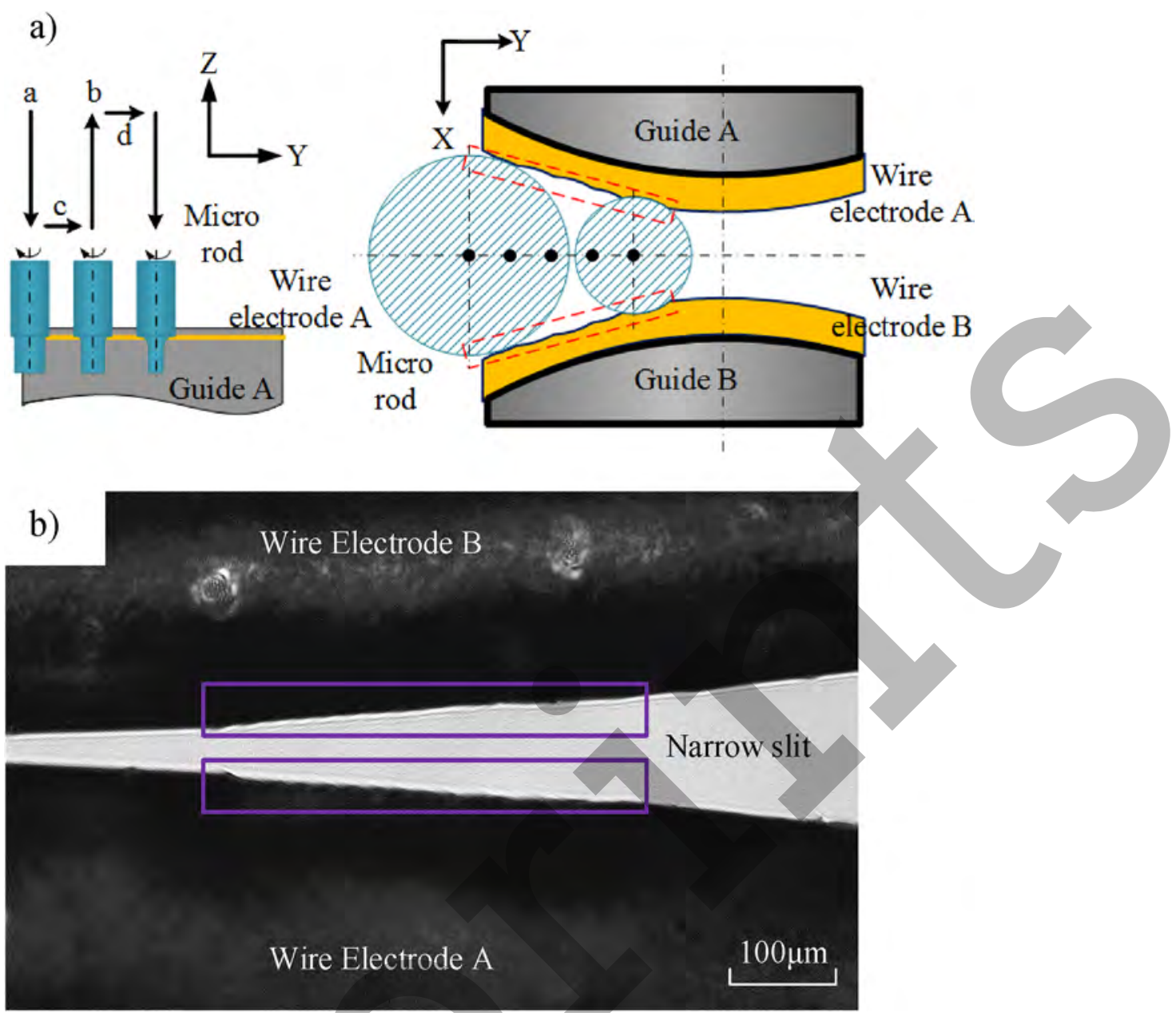

Fig. 10. Surfaces of the twin-wire electrodes after finishing: a) Theoretical analysis of the wear of wire electrode, b) Practical results of wire electrodes wear.

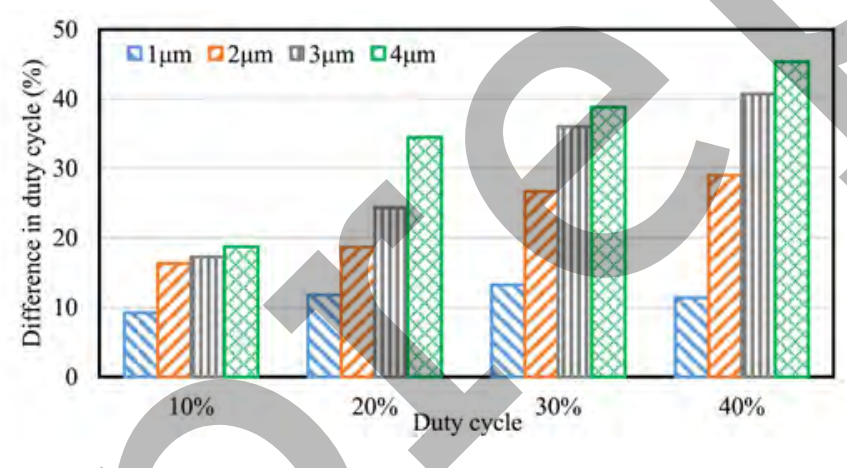

Fig. 11. The effect of the offset distance between the micro rod and the midline on the duty cycle differences.

the duty cycle differences is less than a certain threshold about $5 \%$ $\sim 10 \%$, the micro rod is considered to be located at the midline of the twin wire electrodes.

\section{Results and discussion}

\subsection{Verification of tool setting strategy}

By controlling the position of the micro rod along the tangential direction, the duty cycles $10 \%, 20 \%, 30 \%, 40 \%$ of the tool setting waveform can be obtained. To ensure that the micro rod is located on the symmetry line of the twin-wire electrodes, the position of the micro rod along the $\mathrm{X}$ direction is adjusted to ensure that the duty cycles are equal. Then move the micro rod in the $\mathrm{X}$ direction, so that the offset distances 1 $\mu \mathrm{m}, 2 \mu \mathrm{m}, 3 \mu \mathrm{m}, 4 \mu \mathrm{m}$ between the micro rod and the midline of the twin wires are obtained. Then collect the tool setting voltage signal and count the duty cycle difference of the tool setting waveform at different offset distances. Fig. 11 shows the effect of the offset distance between the micro rod and the midline on the duty cycle differences. It can be seen from the figure that with the increase of the offset distance, the difference of the duty cycles increases gradually. In addition, it also can be seen from the figure that when the offset distance is $1 \mu \mathrm{m}$, the difference in duty cycle of the two-tool setting waveform is $9.2 \%$ when the initial tool setting waveform is $10 \%$. In the process of the tool setting, the duty cycle difference of the tool setting waveform less than $5-10 \%$ can be reached by the position adjustment in X direction. Obviously, the corresponding offset distance will be less than $1 \mu \mathrm{m}$.

The tool setting experiments were carried out. Adjusting the position of the micro rod in the tangential direction, so that the duty cycles of approximately about $30 \%, 40 \%$, and $50 \%$ of the tool setting waveform were obtained. After determining the position of the micro rod, the micro rod machining is performed. During the machining, the wire electrodes remain stationary, so that the tool setting accuracy can be visualized by the wear of the twin-wire electrodes. Fig. 12 is CCD photos showing the wear of the wire electrodes. It can be seen from the figure that the wear of the twin-wire electrodes is the same. This fully proves that the tool-setting strategy can be used to set the position of the micro rod. After applying the tool setting strategy to the new machining method, the micro rod is effectively ensured on the midline of the twinwire electrodes, which can reduce the requirement for the repeated 

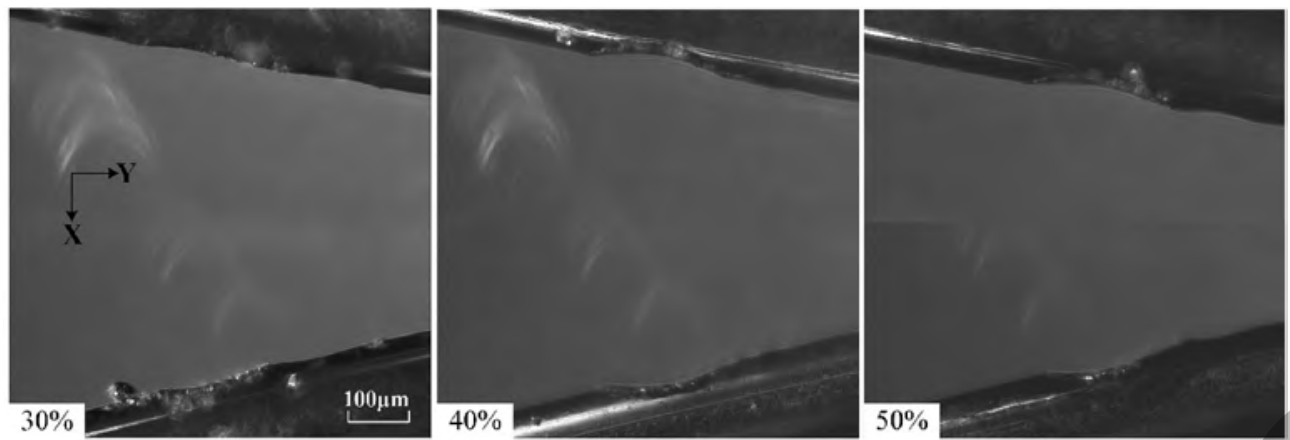

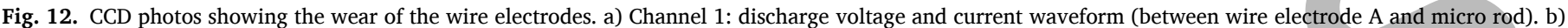
Channel 2: discharge voltage and current waveform (between wire electrode B and micro rod). c) Details of waveforms of discharge voltage and current.

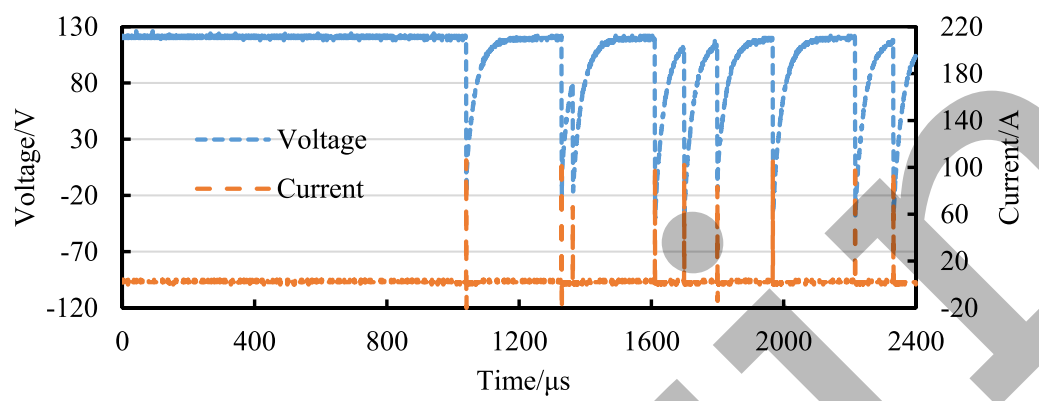

a) Channel 1: discharge voltage and current waveform (between wire electrode $\mathrm{A}$ and micro rod)

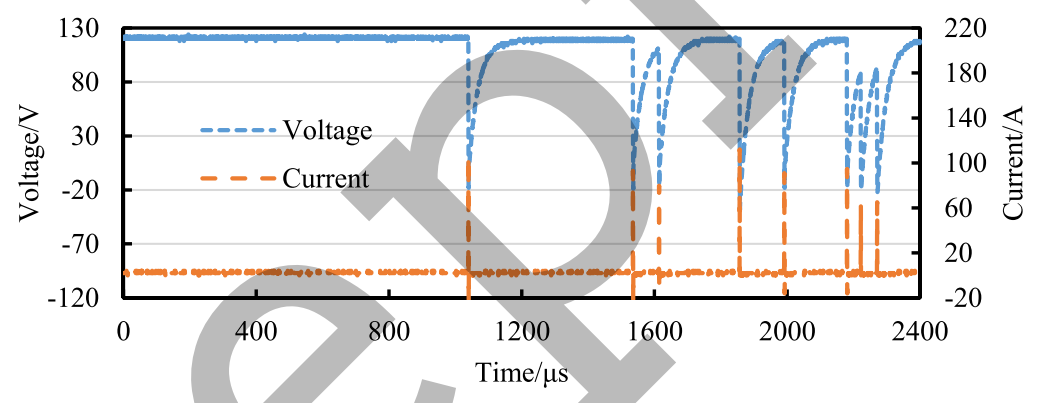

b) Channel 2: discharge voltage and current waveform (between wire electrode B and micro rod)

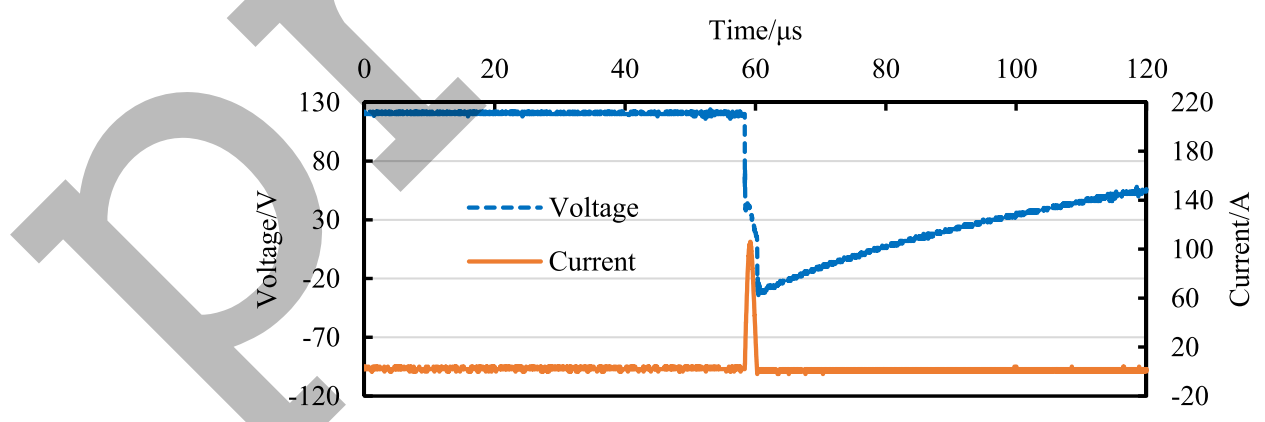

c) Details of waveforms of discharge voltage and current

Fig. 13. Discharge voltage and current waveform during roughing.

\subsection{Discharging process}

The proposed machining method employs two power supplies to synchronously remove material from a micro rod. The key to improve 


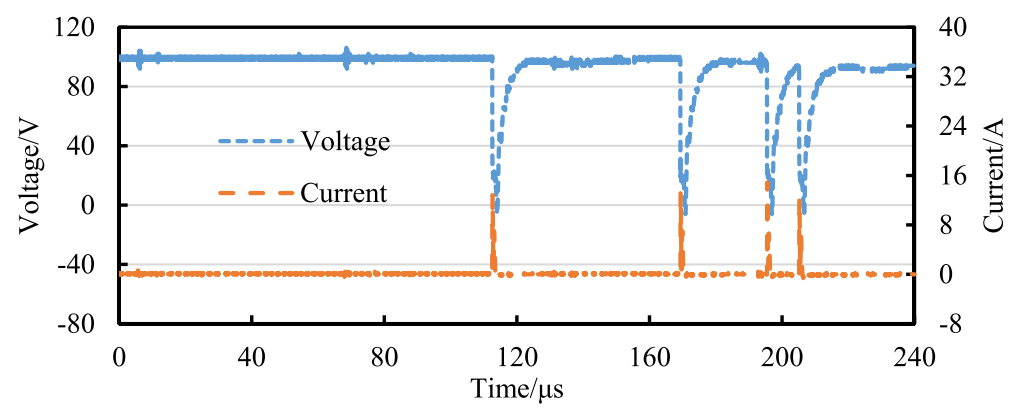

a) Channel 1: discharge voltage and current waveform (between wire electrode $\mathrm{A}$ and micro rod)
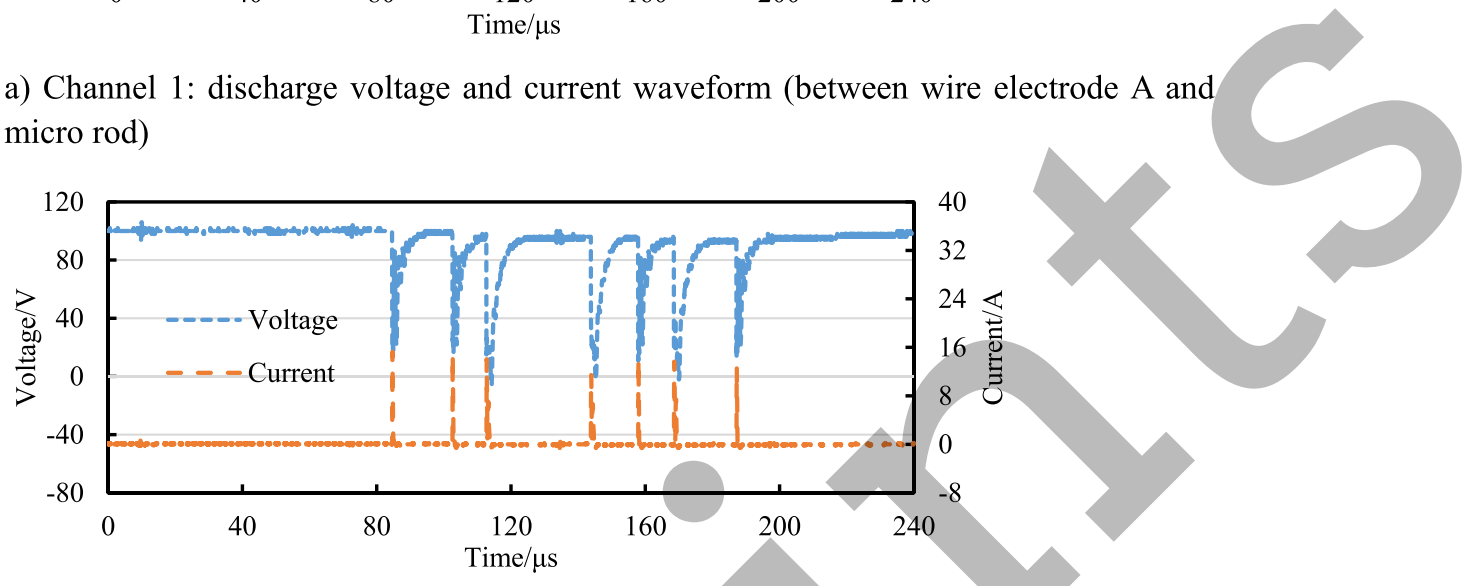

b) Channel 2: discharge voltage and current waveform (between wire electrode B and micro rod)

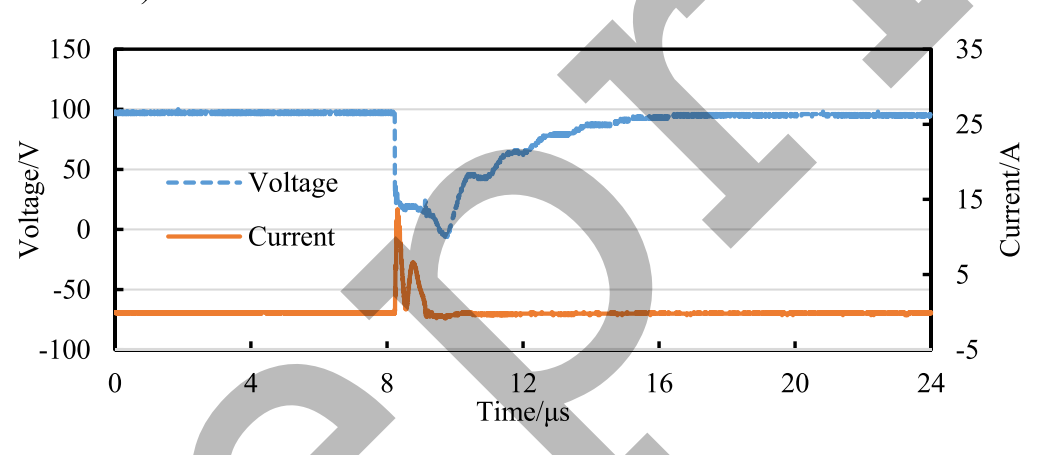

c) Details of waveforms of discharge voltage and current

Fig. 14. Discharge voltage and current waveform during semi-finishing.

the processing efficiency is to ensure that electric discharges are occurring simultaneously between each wire electrode and the processed micro rod. Therefore, observations of the gap voltage waveforms by means of an oscilloscope were performed in order to demonstrate the synchronous occurrence of electric discharges during TMTF-WEDG processing. The discharge parameters of each stage (roughing, semifinishing, and finishing) are listed in Table 1.

In Figs. 13-15, some exemplary gap voltage waveforms during roughing, semi-finishing, and finishing are shown. From these waveforms, it can be observed that electric discharges occur simultaneously between each wire electrode and the micro rod in all the three stages of the machining process. It can be also noticed that the discharges are synchronous, and discharge on both sides may occur at the same time or may be different, because of the application of dual pulse power supply. In roughing, since the applied discharge energy is considerably higher than in semi-finishing and finishing because of the larger open circuit voltage and capacitance. The peak value of discharge current pulse can reach $100 \mathrm{~A}$, but the current pulse width is only about $4 \mu \mathrm{s} \square$ Compared with the discharge time, the charging time of the capacitor is very long. In semi-finishing, as the voltage and capacitance decrease, the peak current decreases to around $16 \mathrm{~A}$. The charging time of the capacitor is significantly shortened, which means that the discharge frequency increases. In finishing, the discharge energy is further reduced because the maximum peak current is only $4 \mathrm{~A}$ and the pulse width is only about 1 $\mu \mathrm{s} \square$

Overall, some differences in terms of shape or duration of the discharge pulses can be observed in the voltage waveforms in Figs. 13-15, even when the discharges are occurring almost synchronously on the two wire electrodes. This is normal since the two RC power supply systems are independent. However, the discharge occurs randomly, which is also a characteristic of the RC mode pulse power supply.

During the experiments, it has also been observed that the wear of the two wire electrodes after TMTF-WEDG processing is substantially the same (as shown in Fig. 10). This further demonstrates that a similar amount of electric discharges occurs between each wire electrode and the micro rod during TMTF-WEDG processing. 


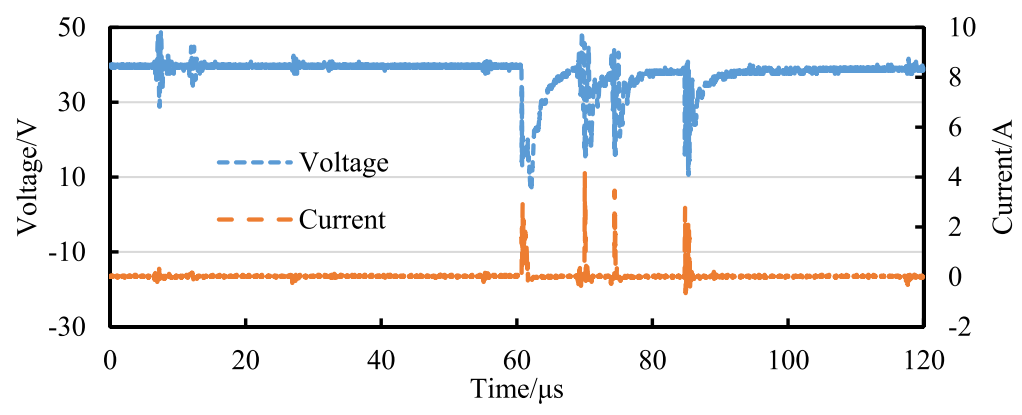

a) Channel 1: discharge voltage and current waveform (between wire electrode $\mathrm{A}$ and micro rod)

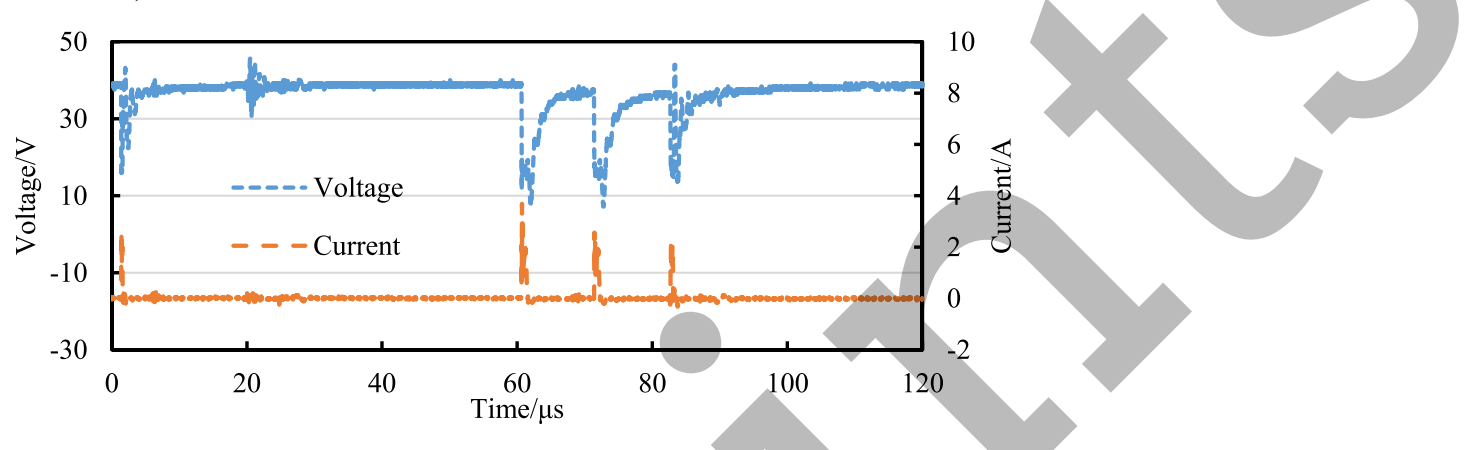

b) Channel 2: discharge voltage and current waveform (between wire electrode B and micro rod)

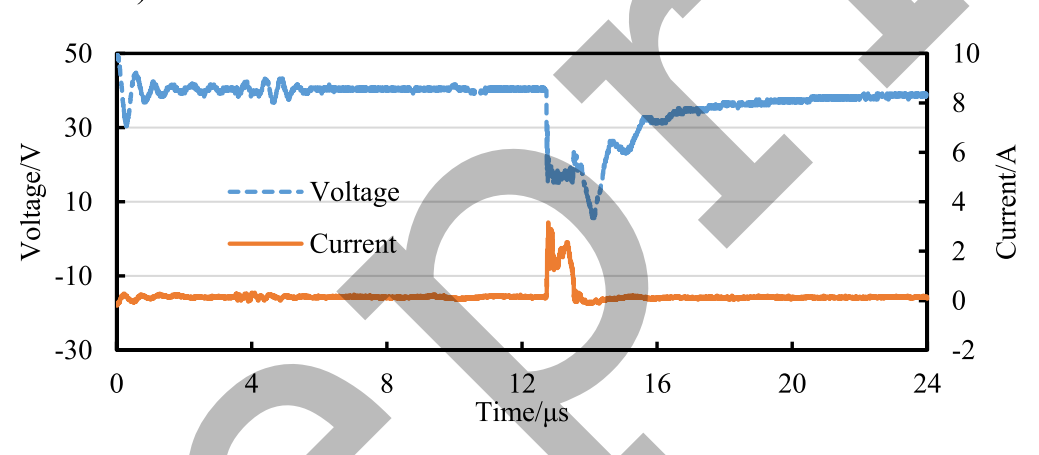

c) Details of waveforms of discharge voltage and current

Fig. 15. Discharge voltage and current waveform during finishing (The experimental parameters are shown in Table 1.).

\subsection{Machining efficiency}

A comparison in terms of machining efficiency of conventional TFWEDG and TMTF-WEDG was carried out. The processing parameters listed in Table 1 were applied during all experiments.

In order to have a meaningful comparison of TF-WEDG and TMTFWEDG methods, the same starting positions were ensured for both processing methods (Fig. 16). In the device used in this research, the position of one wire guide (left wire guide in Fig. 16) can be adjusted to vary the distance $S_{1}$, while the other wire guide is fixed. Therefore, in order to realise TF-WEDG, the distance $S_{1}$ was sufficiently increased by adjusting the position of the movable wire guide, so as to achieve a condition in which discharges only occurred between the micro rod and the wire electrode on the fixed wire guide.

Fig. 17a-b shows the results of the comparison in terms of machining efficiency of TF-WEDG and TMTF-WEDG in roughing. Since the diameter of the micro rods were affected by a certain deviation, the maximum, minimum, and average diameters at each processing position are shown. In Fig. 17a, it can be seen that the processing time to reach a target rod diameter is consistently reduced by TMTF-WEDG. This is due to the effect of the simultaneous discharging occurring between the micro rod and each wire electrode. The deviations in the diameter of the processed micro rods are also reduced by TMTF-WEDG. A detailed comparison of the processing time for some specific experiments is shown in Fig. 17b. For instance, it can be observed that when the target diameter of the micro rod is $50 \mu \mathrm{m}$, the processing time by TMTF-WEDG is $44.78 \mathrm{~min}$, while the processing time by TF-WEDG method is 78.17 min. This means the processing efficiency is increased of about 1.7 times by the proposed TMTF-WEDG method. Similar increase in processing efficiency (i.e. between 1.65 and 1.95 times) can also be observed for the other experiments, which focused on a target dimeter of the micro rods ranging from 30 to $85 \mu \mathrm{m}$. This confirms the significant improvement in processing efficiency by using two wire electrodes and power supply systems.

During the experimental activities, it was also found that the diameter of the micro rods processed by TMTF-WEDG is smaller is smaller than those machined by TF-WEDG with the same tangential feed distance, as shown in Fig. 18. The reason is that when the electric discharges occurs on both wire electrodes, the amount of material removed is larger. This implies that smaller feed distances are needed to obtain 


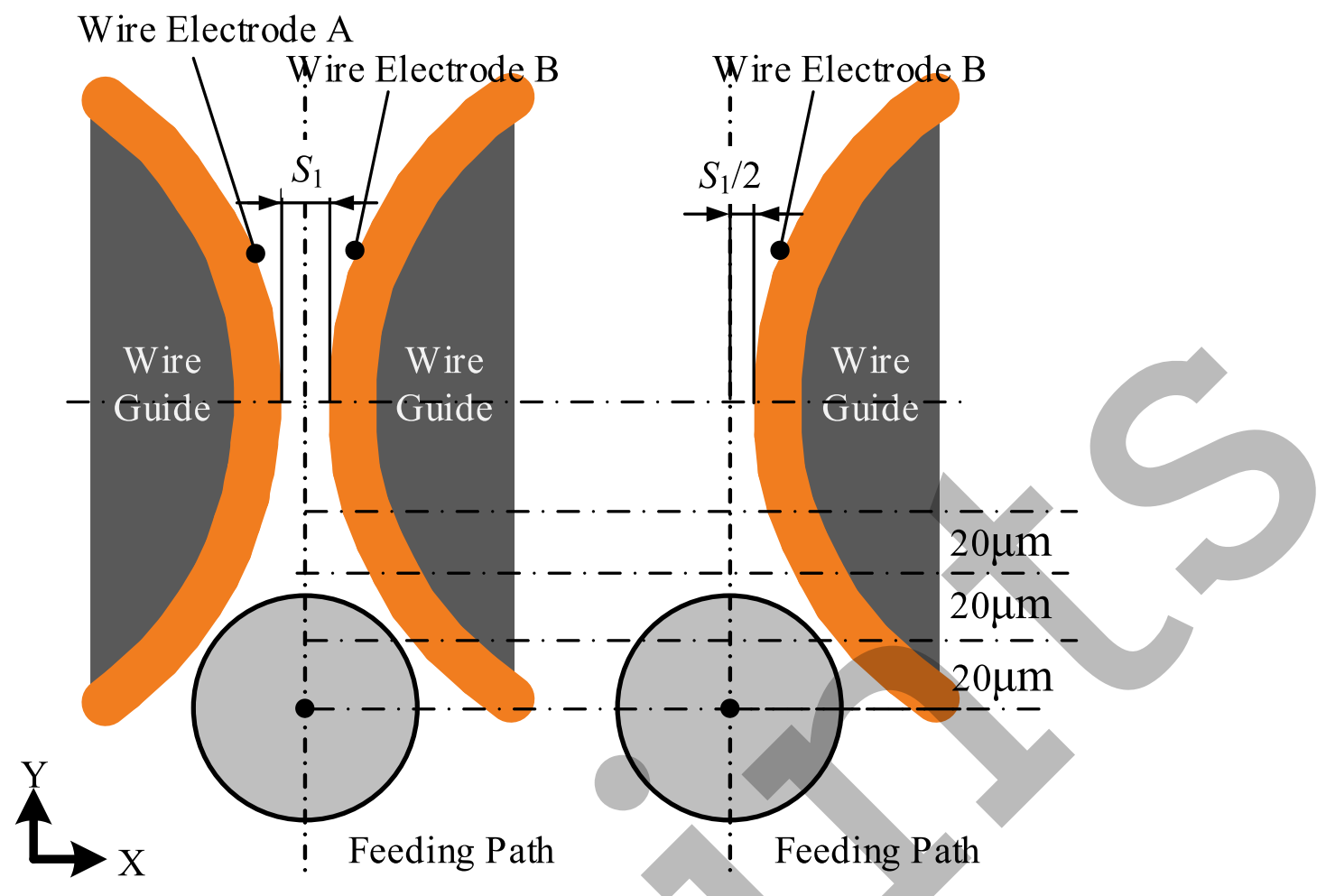

Fig. 16. Initial positions of the micro rod for experimental comparison of TMTF-WEDG (left) and TF-WEDG (right).
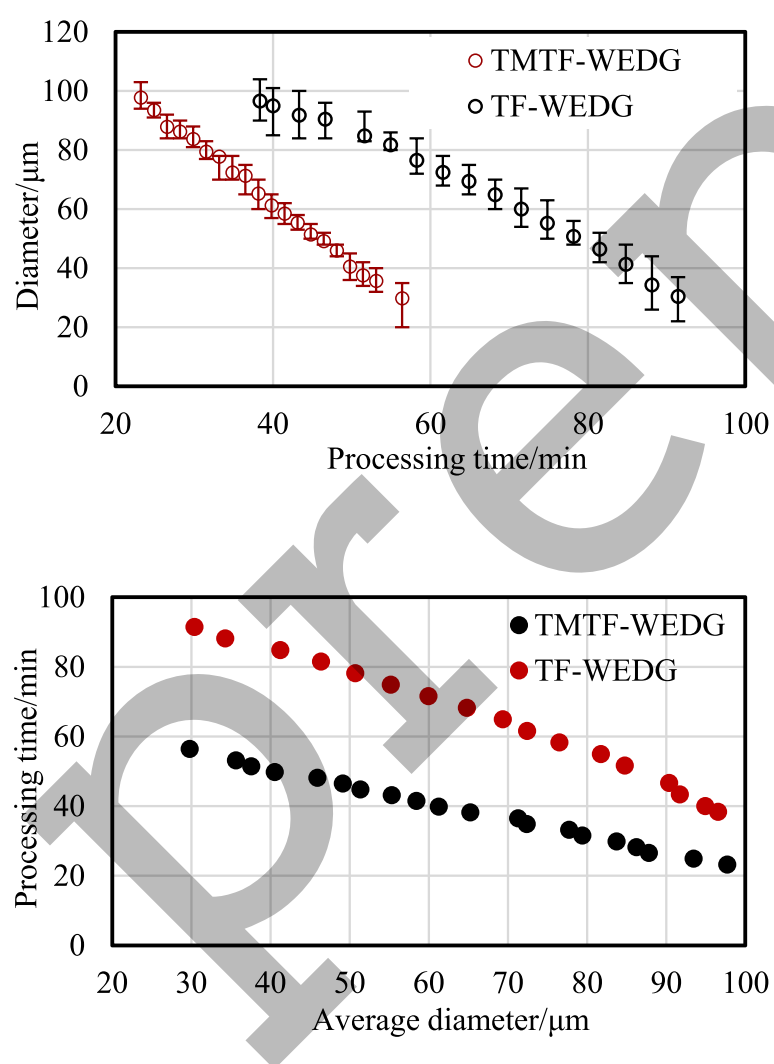

Fig. 17. Machining efficiency comparison of TMTF-WEDG and TF-WEDG in roughing. a) displays diameter vs. time of the micro rods. b) displays the processing time vs. average diameter of the micro rods (The processing parameters listed in Table 1 were applied during all experiments.).

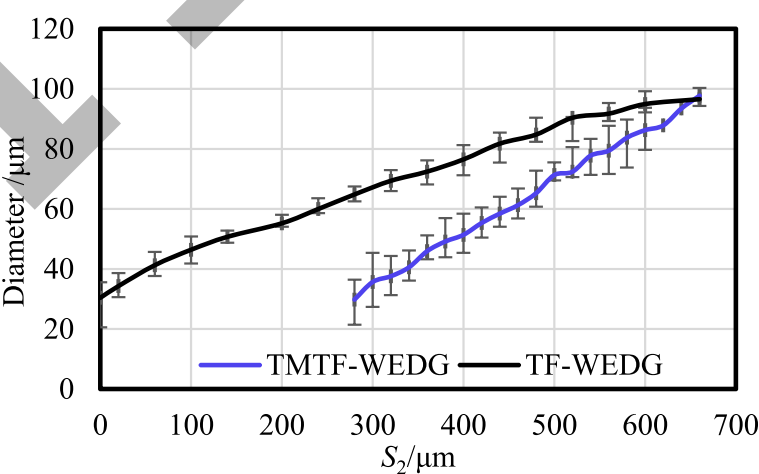

Fig. 18. Reduction in the diameter of the processed micro rods with $S_{2}$ in TMTF-WEDG and TF-WEDG (The processing parameters listed in Table 1 were applied during all experiments.)

the same diameter of a micro rod by TMTF-WEDG than by TF-WEDG. In order to process the micro rods of a certain target diameter, the feed distance required by the TMTF-WEDG is shorter, which improves the processing efficiency.

\subsection{Minimum diameter reduction}

The precision of the TMTF-WEDG process depends on the achievable minimum reduction in the diameter of micro rod. The smaller the reduction in diameter that can be achieved, the easier it is to precisely control the diameter of a micro rod. Micro rods were therefore machined in order to investigate the minimum step in diameter reduction that can be achieved by TMTF-WEDG, and the processing parameters are the parameters used for finishing listed in Table 1.

Fig. 19a-c shows two fabricated micro rods with diameter of $48 \mu \mathrm{m}$ and $71 \mu \mathrm{m}$ respectively. The two micro rods were machined by applying a minimum step in feed direction during the machining process. The enlarged views in Fig. 19-c show the effects on the diameter of the 
a)

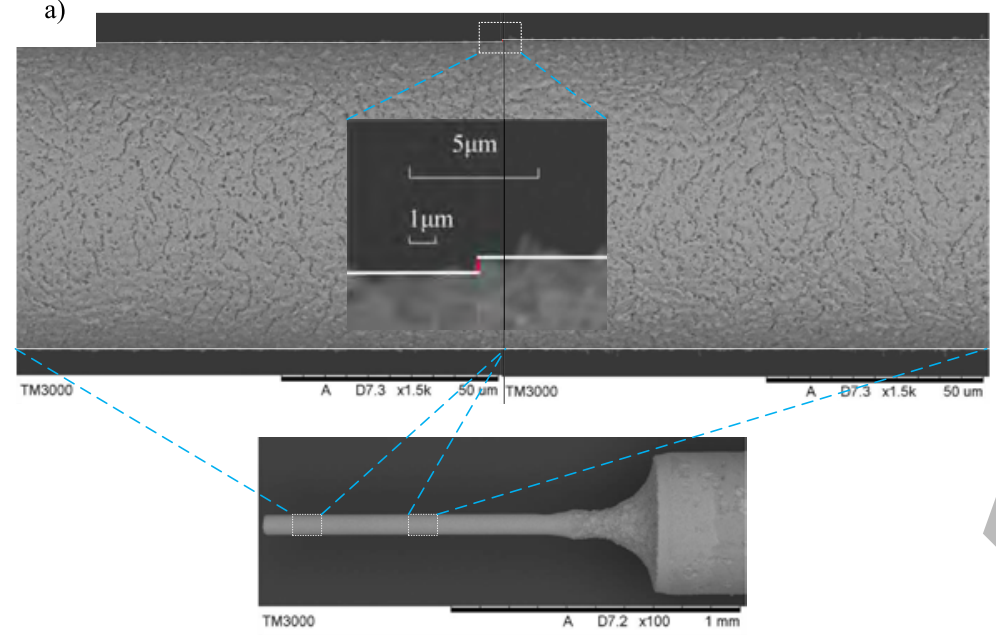

b)

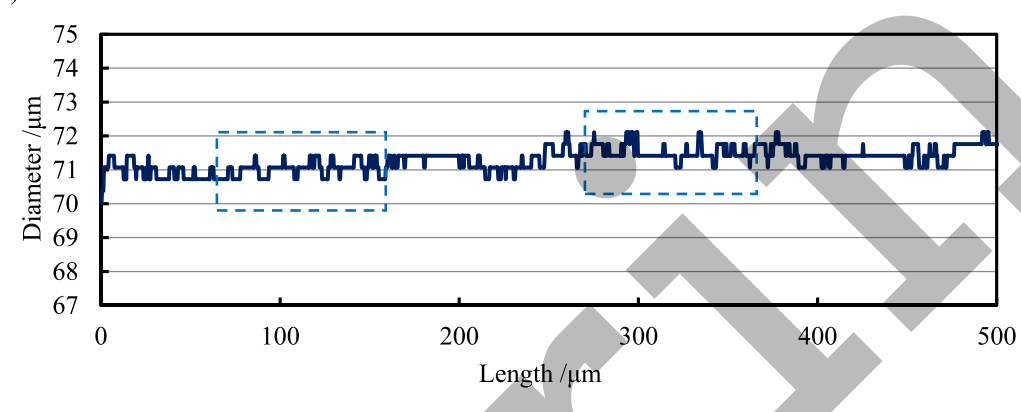

c)
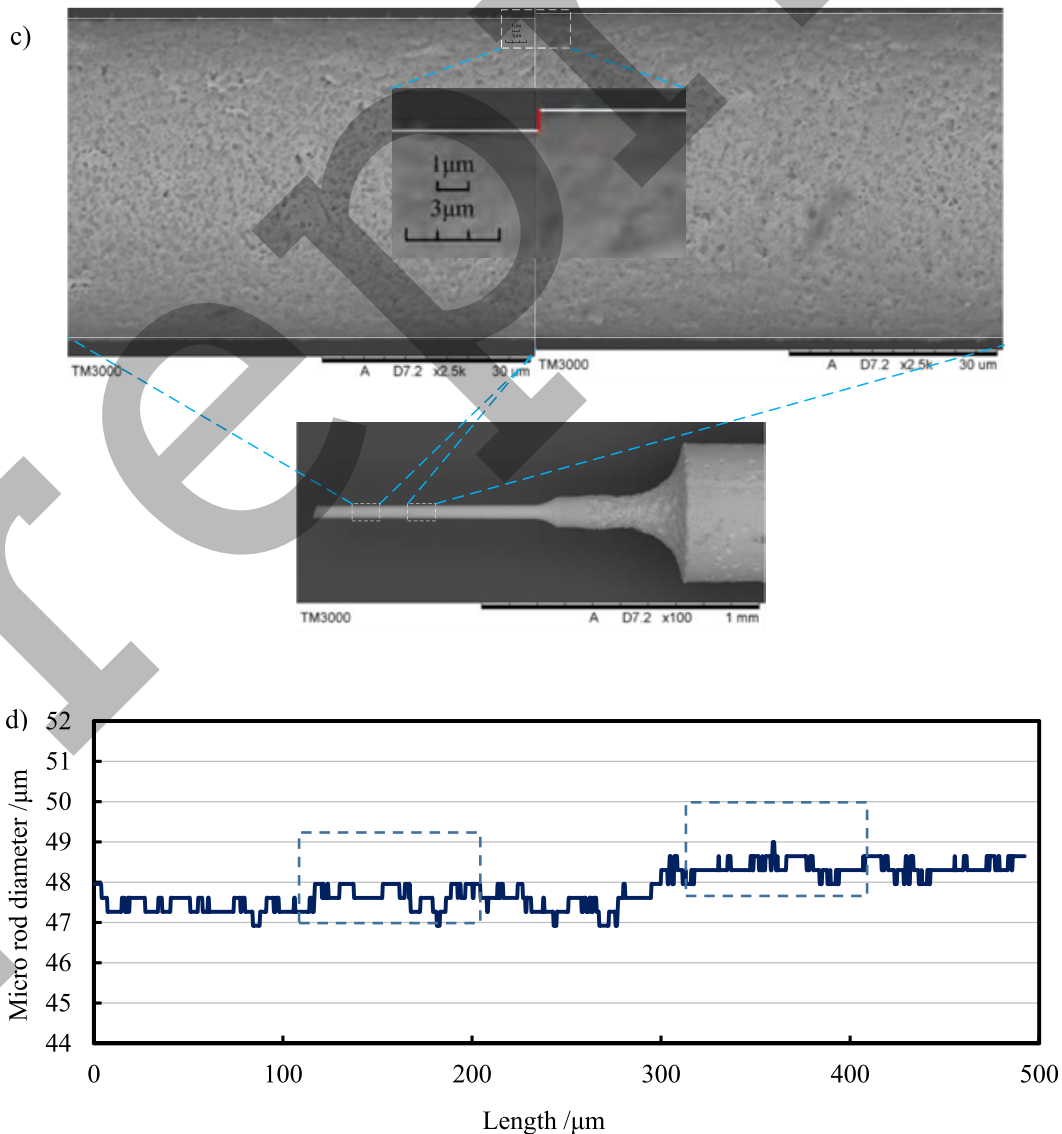

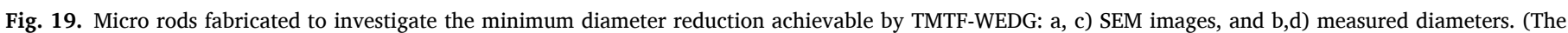
experimental parameters are the finishing parameters listed in Table 1.) 

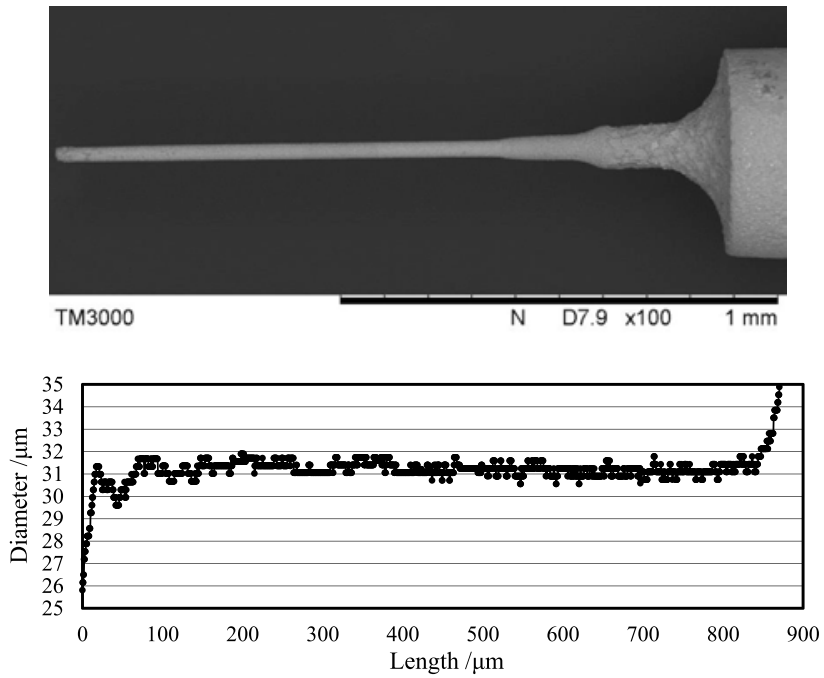

Fig. 20. SEM image and diameter variation of a micro rod machined by TMTFWEDG (The experimental parameters are shown in Table 1.).

machined micro rods. In these figures, the bottom edges of the two sections of the micro rods were aligned to compare the diameter difference. Two white lines were added to facilitate the observation of the differences in diameter. From the measurement results (Fig. 19b-d), it can be concluded that a reduction in diameter of less than $1 \mu \mathrm{m}$ can be realized by varying the feed distance $S_{2}$. For the micro rod shown in Fig. 19a, the increment in feed direction was $2 \mu \mathrm{m}$. The average value of the micro rod diameter is reduced from $71.5 \mu \mathrm{m}$ to $71 \mu \mathrm{m}$. From the micro rod in Fig. 19c, the increment in feed direction was $4 \mu \mathrm{m}$. The average diameter of the micro rod was reduced from $48.3 \mu \mathrm{m}$ to 47.6 $\mu \mathrm{m}$.

\subsection{Machining accuracy and repeatability}

Further experiments were performed to investigate the accuracy and repeatability in machining micro rods by TMTF-WEDG with the parameters listed in Table 1. Fig. 20 shows a SEM image and the diameter variation of a micro rod fabricated by TMTF-WEDG. The average diameter of the micro rod is $31 \mu \mathrm{m}$. It can be seen that the diameter variation is limited within $1 \mu \mathrm{m}$ in a length range of $800 \mu \mathrm{m}$.

Fig. 21a-e shows five micro rods fabricated to study the repeatability of the TMTF-WEDG process. The target diameter value was $45 \mu \mathrm{m}$. As for the micro rod in Fig. 21, the measurement results (Fig. 21f) confirm that the diameter variation of a single micro rod can be controlled within 1
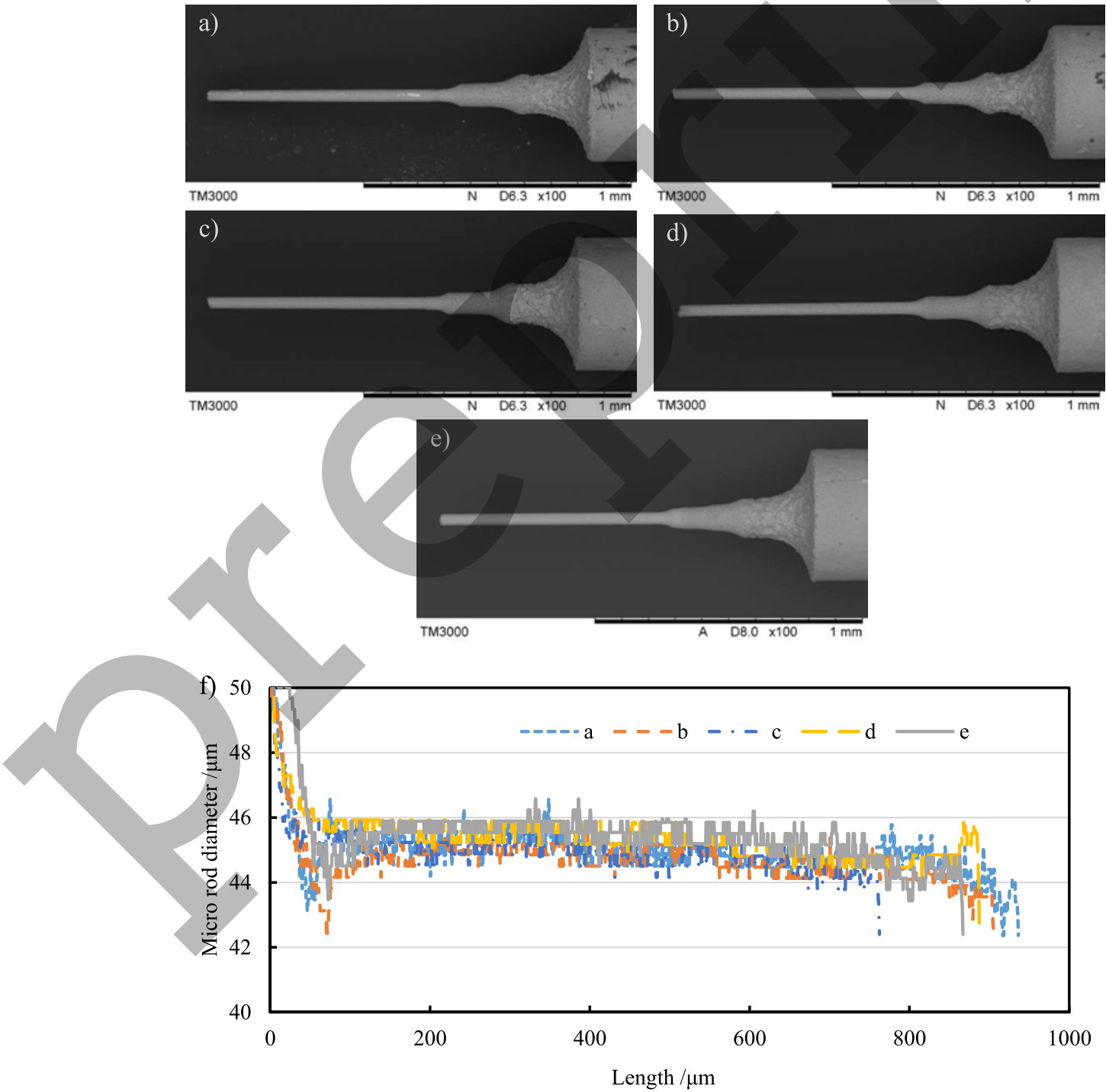

Fig. 21. a-e) SEM images of the five micro rods fabricated by TMTF-WEDG and f) respective diameters (The experimental parameters are shown in Table 1.). 
$\mu \mathrm{m}$ in the length range of $800 \mu \mathrm{m}$. Moreover, it can be noticed that the variations in diameter of the five micro rods are within less than $2 \mu \mathrm{m}$. This demonstrates that TMTF-WEDG is capable of achieving a similar if not higher machining accuracy and repeatability of already existing electrical discharge grinding techniques, such as TF-WEDG [8] and EDG-TBE [10].

\section{Conclusions}

Focusing on simplifying micro rod diameter control strategies and improving the accuracy and efficiency in machining cylindrical micro rods, the TMTF-WEDG method was proposed in this paper. A theoretical analysis of the machining characteristics of this method was illustrated. Then, experimental activities were presented to demonstrate the feasibility and capability of the proposed method. Based on the experimental results, the following conclusions can be drawn:

- In TMTF-WEDG, the diameter of the micro rods can be controlled by the tangential feed strategy and the narrow slit formed by the mirrorsymmetrical twin-wire electrodes in the same plane. The width of the narrow slit varies nonlinearly with the tangential feed, which allows the diameter of the micro rods to be controlled by controlling the tangential feed distance.

- Compared to conventional TF-WEDG, in which a single wire electrode is used, TMTF-WEDG can improve the processing efficiency in machining a cylindrical micro rod by about 1.7 times. Moreover, at the same tangential position, the diameter of the micro rods fabricated by TMTF-WEDG is typically smaller than those fabricated by TF-WEDG.

- A cutting depth in radial direction of less than $1 \mu \mathrm{m}$ can be obtained by TMTF-WEDG, which indicates that high-resolution material removal can be obtained. This is a crucial factor in order to achieve a high degree of accuracy and repeatability of the machining process.

- A tolerance of less than $1 \mu \mathrm{m}$ can be achieved on the diameter of micro rods manufactured by TMTF-WEDG of $800 \mu \mathrm{m}$ length and $<50$ $\mu \mathrm{m}$ diameter. The repeatability of the machining process is below 2 $\mu \mathrm{m}$.

\section{Declaration of competing interest}

The authors declare that they have no known competing financial interests or personal relationships that could have appeared to influence the work reported in this paper.

\section{Acknowledgments}

This research is supported by the National Natural Science Foundation of China of China (Grant No. 51605323) and Key Research and Development Program of Shanxi Province (Grant No. 201703D121006).

\section{References}

[1] Deepak K, Nirmal KS, Vivek B. Recent trends, opportunities and other aspects of micro-EDM for advanced manufacturing: a comprehensive review. J Braz Soc Mech Sci Eng 2020;42(5).

[2] Singh SK, Mali HS. Microfeatures and microfabrication: current role of microelectric discharge machining. J Micromech Microeng 2019;29(4).

[3] Pham DT, Dimov SS, Bigot S, et al. Micro EDM-recent developments and research issues. J Mater Process Technol 2004;149(1):50-7.

[4] Koyano T, Sugata Y, Hosokawa A, Furumoto T. Micro-electrical discharge machining of micro-rods using tool electrodes with high electrical resistivity. Precis Eng 2019;55:95-100.

[5] Mehdi H, Ahmed ADS, Noordin MY. Proposing new fabrication and measurement techniques of micro-electrodes with high aspect ratio for micro EDM drilling of tungsten carbide (WC) using EDM machine. Measurement 2017;97:64-78.

[6] Masuzawa T, Fújino M, Kobayashi K, et al. Wire electro-discharge grinding for micro-machining. CIRP Ann - Manuf Technol 1985;34(1):431-4.

[7] Zhang L, Tong H, Li Y. Precision machining of micro tool electrodes in micro EDM for drilling array micro holes. Precis Eng 2015;39:100-6.

[8] Li Z, Bai J, Cao Y, et al. Fabrication of microelectrode with large aspect ratio and precision machining of micro-hole array by micro-EDM. J Mater Process Technol 2019;268:70-9.

[9] Wang YQ, Bai JC. Diameter control of micro shafts in wire electrical discharge grinding. Int J Adv Manuf Technol 2014;72(9-12):1747-57.

[10] Qingfeng Y, Xingqiao W, Ping W, et al. Fabrication of micro rod electrode by electrical discharge grinding using two block electrodes. J Mater Process Technol 2016;234:143-9.

[11] Mohri Naotake, Takayuki. Micro-pin electrodes formation by micro-scanning EDM process. CIRP Ann - Manuf Technol 2006;55:175-8. 01.

[12] Sheu DY. Gradation twin-wire EDM manufacturing system. U.S. Patent 2011;7 (906):3-15. 743.

[13] Sheu DY. Microelectrode tools manufacturing by hybrid circuits twin-wire electro discharge grinding. Mater Manuf Process 2010;25(10):1142-7. 